\title{
Polynomial Invariants and Harmonic Functions Related to Exceptional Regular Polytopes
}

\author{
Katsunori Iwasaki, Atsufumi Kenma, and Keiji Matsumoto
}

\section{CONTENTS}

1. Introduction

2. Main Theorem

3. Invariant Theory

4. Canonical Invariant Bases

5. Mean Value Problem

References

2000 AMS Subject Classification: Primary 52 B 11; Secondary 20 F 55

Keywords: Polynomial invariants, harmonic functions, exceptional regular polytopes, mean value property, finite reflection groups
We compute certain polynomial invariants for the finite reflection groups of the types $H_{3}, H_{4}$ and $F_{4}$. Using this result, we explicitly determine the solution space of functions satisfying a mean value property related to the exceptional regular polytopes, namely, the icosahedron and dodecahedron in three dimensions and the 24-cell, 600-cell, and 120-cell in four dimensions.

\section{INTRODUCTION}

A classical theorem of Gauss and Koebe states that a function is harmonic if and only if it satisfies the mean value property with respect to a sphere. In this paper, we study the following variant of this property for polytopes.

Given an $n$-dimensional polytope $P$ and an integer $k \in\{0,1, \ldots, n\}$, let $P(k)$ be the $k$-dimensional skeleton of $P$. An $\mathbb{R}$-valued continuous function $f \in C\left(\mathbb{R}^{n}\right)$ is said to be $P(k)$-harmonic if it satisfies the mean value property:

$$
f(x)=\frac{1}{|P(k)|} \int_{P(k)} f(x+r y) d \mu_{k}(y)
$$

for any $x \in \mathbb{R}^{n}$ and $r>0$, where $\mu_{k}$ is the $k$-dimensional volume element on $P(k)$ and $|P(k)|=\mu_{k}(P(k))$ is the $k$-dimensional total mass of $P(k)$. Let $\mathcal{H}_{P(k)}$ denote the set of $P(k)$-harmonic functions. We are interested in the problem of characterizing the function space $\mathcal{H}_{P(k)}$.

From our previous work [Iwasaki 97a], the following facts are known: The space $\mathcal{H}_{P(k)}$ is a finite-dimensional linear space of polynomials. The space $\mathcal{H}_{P(k)}$ is invariant under partial differentiations, namely, it carries a structure of $\mathbb{R}[\partial]$-module, where $\mathbb{R}[\partial]$ is the ring of partial differential operators with constant coefficients. If the symmetry group $G \subset O(n)$ of $P$ is irreducible, then $\mathcal{H}_{P(k)}$ is a finite-dimensional linear space of harmonic polynomials.

Our problem is of particular interest when the polytope is a regular convex polytope. Now we recall the 


\begin{tabular}{|c|c|c|}
\hline type & polytope & dimension \\
\hline$A_{n}$ & regular simplex, self dual & $n \geq 3$ \\
\hline$B_{n}$ & cross polytope and measure polytope & $n \geq 3$ \\
\hline$F_{4}$ & 24-cell, self dual & $n=4$ \\
\hline$H_{3}$ & icosahedron and dodecahedron & $n=3$ \\
\hline$H_{4}$ & 600-cell and 120 -cell & $n=4$ \\
\hline$I_{2}(m)$ & regular convex $m$-gon, self dual & $n=2$ \\
\hline
\end{tabular}

TABLE 1. Classification of regular convex polytopes.

classification of regular convex polytopes in terms of their symmetry groups (see [Coxeter 73]). The symmetry groups of the regular convex polytopes are the irreducible finite reflection groups of types $A_{n}, B_{n}, F_{4}$, $H_{3}, H_{4}$, and $I_{2}(m)$ (see [Humphreys 90]). The correspondence between the polytopes and the types is given in Table 1. Observe that certain types, e.g., $H_{3}$, correspond to two polytopes, which are duals of each other. Polytopes of the types $H_{3}, F_{4}$, and $H_{4}$ are called the $e x$ ceptional regular polytopes, as they appear sporadically in the classification.

For any $n$-dimensional regular convex polytope $P$, one has $\mathcal{H}_{P(n-1)}=\mathcal{H}_{P(n)}$ by [Iwasaki 97 a, Theorem 2.2]. Hence it is sufficient to consider the $k$-skeleton problem for $k \in\{0,1, \ldots, n-1\}$. For each regular convex polytope, the 0 -skeleton problem, or the vertex problem, was thoroughly discussed by many authors [Kakutani and Nagumo 35, Walsh 36, Beckenbach and Reade 43, Beckenbach and Reade 45, Friedman 1957, Flatto 63, Flatto and Wiener 70, Haeuslein 70]. Our main concern is the much more involved higher-skeleton problems. In this direction, Flatto [Flatto 63] solved the $(n-1)$-skeleton problem for a regular $n$-simplex and an $n$-dimensional cross polytope. However, attempts to deal with every skeleton have begun only recently. Iwasaki [Iwasaki 97b] settled the problem for all skeletons of a regular $n$ simplex. This paper focuses on the same problem for the exceptional regular polytopes and gives a complete solution to it. The remaining polytopes will be discussed elsewhere.

To obtain our result, we employ a criterion established in an earlier paper [Iwasaki 99a] by one of the authors. See (3-4) of Theorem 3.2. The new material of the present paper consists of elaborate computations needed to verify the criterion. For this purpose, we used the computer algebra system, Maple.

\section{MAIN THEOREM}

We take the icosahedron, dodecahedron, 24-cell, 600-cell, and 120-cell in such a manner that their vertices are as

\begin{tabular}{|c|l|}
\hline polytope & \multicolumn{1}{|c|}{ coordinates of vertices } \\
\hline $\begin{array}{c}\text { Icosahedron }\{3,5\} \\
\text { Dodecahedron } \\
\{5,3\}\end{array}$ & $(0, \pm \tau, \pm 1),( \pm 1,0, \pm \tau),( \pm \tau, \pm 1,0)$ \\
$( \pm 1, \pm 1, \pm 1)$
\end{tabular}

TABLE 2. Vertices of the exceptional regular polytopes.

in Table 2, where $\tau$ stands for the golden ratio:

$$
\tau=\frac{1+\sqrt{5}}{2}
$$

These polytopes are expressed as $\{3,5\},\{5,3\},\{3,4,3\}$, $\{3,3,5\},\{5,3,3\}$, respectively, in Schläfli's symbols. Here $\{p, q\}$ represents a regular polyhedron whose faces are regular $p$-gons and whose vertex figures are regular $q$-gons, while $\{p, q, r\}$ represents a 4 -dimensional regular polytope whose cells are $\{p, q\} \mathrm{s}$ and whose vertex figures are $\{q, r\}$ s (see [Coxeter 73]). Note that $\{3,5\}$ and $\{5,3\}$ (respectively $\{3,3,5\}$ and $\{5,3,3\}$ ) are duals of each other.

For each $\sharp=H_{3}, F_{4}, H_{5}$, let $G_{\sharp}$ be a finite reflection group of type $\sharp$ realized as the symmetry group of $\{3,5\}$, $\{3,4,3\},\{3,3,5\}$, respectively. The groups $G_{H_{3}}$ and $G_{H_{4}}$ are also realized as the symmetry groups of $\{5,3\}$ and $\{5,3,3\}$, respectively. Then the fundamental alternating polynomial $\Delta_{\sharp}=\Delta_{G_{\sharp}}$ of the group $G_{\sharp}$ is given in Table 3 , where the notation $\prod$ is used in the following sense:

$$
\begin{aligned}
& \prod\left(a_{0} \pm a_{1} \pm \cdots \pm a_{m}\right) \\
& \quad=\prod_{\varepsilon_{1}= \pm 1} \cdots \prod_{\varepsilon_{m}= \pm 1}\left(a_{0}+\varepsilon_{1} a_{1}+\cdots+\varepsilon_{m} a_{m}\right) .
\end{aligned}
$$

The reflecting hyperplanes of the reflection group $G_{\sharp}$ are given by the locus of the equation $\Delta_{\sharp}=0$. Moreover, the order of the group $G_{\sharp}$ is given by

$$
\left|G_{\sharp}\right|=\left\{\begin{aligned}
120 & \left(\sharp=H_{3}\right), \\
1152 & \left(\sharp=F_{4}\right), \\
14400 & \left(\sharp=H_{4}\right) .
\end{aligned}\right.
$$

The main theorem of the present paper is now stated as follows: 


$\begin{array}{|rl|}\Delta_{H_{3}}=\quad x y z \quad & \prod\left(\tau x \pm \tau^{-1} y \pm z\right) \prod\left(\tau y \pm \tau^{-1} z \pm x\right) \prod\left(\tau z \pm \tau^{-1} x \pm y\right) \\ \Delta_{F_{4}}=x y z w \quad & \prod(x \pm y \pm z \pm w) \\ & \prod(x \pm y) \prod(x \pm z) \prod(x \pm w) \\ & \prod(y \pm z) \prod(y \pm w) \prod(z \pm w) \\ \Delta_{H_{4}}=x y z w & \prod(x \pm y \pm z \pm w) \\ & \prod\left(\tau x \pm \tau^{-1} y \pm z\right) \prod\left(\tau y \pm \tau^{-1} z \pm x\right) \prod\left(\tau z \pm \tau^{-1} x \pm y\right) \\ & \prod\left(\tau x \pm \tau^{-1} z \pm w\right) \prod\left(\tau z \pm \tau^{-1} w \pm x\right) \prod\left(\tau w \pm \tau^{-1} x \pm z\right) \\ & \prod\left(\tau x \pm \tau^{-1} w \pm y\right) \prod\left(\tau w \pm \tau^{-1} y \pm x\right) \prod\left(\tau y \pm \tau^{-1} x \pm w\right) \\ & \prod\left(\tau y \pm \tau^{-1} w \pm z\right) \prod\left(\tau w \pm \tau^{-1} z \pm y\right) \prod\left(\tau z \pm \tau^{-1} y \pm w\right)\end{array}$

TABLE 3. Fundamental alternating polynomials.

Theorem 2.1. Let $P$ be an $n$-dimensional exceptional regular convex polytope $(n=3$ or 4$)$ centered at the origin and let $G$ be its symmetry group. Then for each $k \in\{0,1, \ldots, n\}$, the fundamental alternating polynomial $\Delta_{G}$ of the reflection group $G$ generates the function space $\mathcal{H}_{P(k)}$ as an $\mathbb{R}[\partial]$-module, namely,

$$
\mathcal{H}_{P(k)}=\mathbb{R}[\partial] \Delta_{G}
$$

In particular, the space $\mathcal{H}_{P(k)}$ is independent of the skeletons of $P$. The dimension of $\mathcal{H}_{P(k)}$ is the order $|G|$ of the group $G$, that is,

$$
\operatorname{dim} \mathcal{H}_{P(k)}=|G| .
$$

\section{INVARIANT THEORY}

The proof of Theorem 2.1 is based on some results in invariant theory established by Iwasaki [Iwasaki 97c, Iwasaki 99a]. Let $G$ be a finite reflection group acting on $\mathbb{R}^{n}$. The ring of $G$-invariant polynomials in $\mathbb{R}[x]=\mathbb{R}\left[x_{1}, \ldots, x_{n}\right]$ is generated by an $n$-tuple of algebraically independent homogeneous $G$-invariant polynomials. Such an $n$-tuple $\left(\phi_{1}, \ldots, \phi_{n}\right)$ is called an invariant basis for $G$, where $\phi_{1}, \ldots, \phi_{n}$ are arranged so that the degrees $d_{i}=\operatorname{deg} \phi_{i}(i=1, \ldots, n)$ satisfy $d_{1} \leq \cdots \leq d_{n}$. The degrees $\left(d_{1}, \ldots, d_{n}\right)$ depend only on $G$, that is, independent of a particularly chosen invariant basis. An invariant basis $\left(\phi_{1}, \ldots, \phi_{n}\right)$ is said to be canonical if it satisfies the system of nonlinear partial differential equations:

$$
\phi_{i}(\partial) \phi_{j}=\left\langle\phi_{i}, \phi_{j}\right\rangle \delta_{i j} \quad(i, j=1, \ldots, n),
$$

where $\langle f, g\rangle$ is an inner product on $\mathbb{R}[x]$ defined by

$$
\langle f, g\rangle=\left.f(\partial) g\right|_{x=0} \quad(f, g \in \mathbb{R}[x]),
$$

and $\delta_{i j}$ is Kronecker's symbol. From a result of [Iwasaki 97c], any finite reflection group admits a canonical invariant basis, which is unique in the following sense: if $\left(\phi_{1}, \ldots, \phi_{n}\right)$ and $\left(\psi_{1}, \ldots, \psi_{n}\right)$ are two canonical invariant bases, then $\phi_{1}, \ldots, \phi_{n}$ are linear combinations of $\psi_{1}, \ldots, \psi_{n}$ and vice versa. In particular, if the degrees $\left(d_{1}, \ldots, d_{n}\right)$ satisfy $d_{1}<\cdots<d_{n}$, then for each $i \in\{1, \ldots, n\}$ the $i$-th canonical invariant polynomial $\phi_{i}$ is unique up to a nonzero constant multiple. The canonical invariant bases of the types $A_{n}, B_{n}, D_{n}$, and $I_{2}(m)$ were explicitly calculated in [Iwasaki 97c]. Other results from our previous work that will be used in Sections 4 and 5 include:

Theorem 3.1. ([Iwasaki 97c]) Let $\left(\psi_{1}, \ldots, \psi_{n}\right)$ be an orthogonal invariant basis for $G$ relative to the inner product (3-1). Then the system of partial differential equations

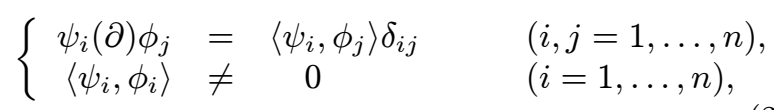

admits a solution $\left(\phi_{1}, \ldots, \phi_{n}\right)$ such that each $\phi_{i}$ is a $G$ invariant smooth function on $\mathbb{R}^{n}$ with $\phi_{i}(0)=0$. Moreover, any such solution $\left(\phi_{1}, \ldots, \phi_{n}\right)$ of (3-2) is a canonical invariant basis for $G$.

Theorem 3.2. [Iwasaki 99a] Let $P$ be an $n$-dimensional polytope having a finite reflection group $G$ as its symmetry group. Assume that the degrees $\left(d_{1}, \ldots, d_{n}\right)$ of $G$ satisfy the condition

$$
d_{1}<d_{2}<\cdots<d_{n}
$$

and let $\left(\phi_{1}, \ldots, \phi_{n}\right)$ be the canonical invariant basis for $G$. Then for each $k \in\{0,1, \ldots, n\}$, the fundamental alternating polynomial $\Delta_{G}$ of the group $G$ generates the 
function space $\mathcal{H}_{P(k)}$ as an $\mathbb{R}[\partial]$-module and the dimension of $\mathcal{H}_{P(k)}$ is the order $|G|$ of $G$, if and only if $P(k)$ satisfies

$$
\int_{P(k)} \phi_{i}(x) d \mu_{k}(x) \neq 0 \quad(i=1, \ldots, n) .
$$

In Section 4, we will apply Theorem 3.1 to compute the canonical invariant bases for the groups $G_{\sharp}$ with $\sharp=$ $H_{3}, F_{4}, H_{4}$. In Section 5 , we will verify the criterion (3-4) of Theorem 3.2 to establish Theorem 2.1.

\section{CANONICAL INVARIANT BASES}

For each $\sharp=H_{3}, F_{4}, H_{4}$, we shall explicitly compute the canonical invariant basis for the group $G_{\sharp}$. Recall that the degrees of $G_{\sharp}$ are given by

$$
\begin{aligned}
(2,6,10) & \left(\sharp=H_{3}\right), \\
(2,6,8,12) & \left(\sharp=F_{4}\right), \\
(2,12,20,30) & \left(\sharp=H_{4}\right) .
\end{aligned}
$$

To state the result, we establish some notation. Given a partition $\lambda=\left(\lambda_{1}, \ldots, \lambda_{n}\right)$ with $\lambda_{1} \geq \cdots \geq \lambda_{n} \geq 0$, let $M_{\lambda}$ denote the associated monomial symmetric polynomial of the variables $\left(x_{1}^{2}, \ldots, x_{n}^{2}\right)$, namely,

$$
M_{\lambda}=\sum x_{1}^{2 \mu_{1}} \cdots x_{n}^{2 \mu_{n}}
$$

where the sum is taken over all permutations $\left(\mu_{1}, \ldots, \mu_{n}\right)$ of $\left(\lambda_{1}, \ldots, \lambda_{n}\right)$. If $\lambda$ consists of mutually distinct numbers $p_{1}>\cdots>p_{m}$ with $p_{j}$ appearing $k_{j}$ times in $\lambda$, then we put

$$
M_{\lambda}=\left[p_{1}^{k_{1}}|\cdots| p_{m}^{k_{m}}\right] .
$$

For example, if $\lambda=(1,0,0),(2,1,0),(1,1,1)$, then

$$
\begin{aligned}
{\left[1 \mid 0^{2}\right] } & =x_{1}^{2}+x_{2}^{2}+x_{3}^{2}, \\
{[2|1| 0] } & =x_{1}^{4} x_{2}^{2}+x_{2}^{4} x_{3}^{2}+x_{3}^{4} x_{1}^{2}+x_{1}^{2} x_{2}^{4}+x_{2}^{2} x_{3}^{4}+x_{3}^{2} x_{1}^{4}, \\
{\left[1^{3}\right] } & =x_{1}^{2} x_{2}^{2} x_{3}^{2} .
\end{aligned}
$$

Moreover, let $\Delta_{n}$ be the fundamental alternating polynomial of $\left(x_{1}^{2}, \ldots, x_{n}^{2}\right)$ :

$$
\Delta_{n}=\prod_{1 \leq i<j \leq n}\left(x_{i}^{2}-x_{j}^{2}\right) .
$$

Theorem 4.1. For each $\sharp=H_{3}, F_{4}, H_{4}$, the canonical invariant basis for the group $G_{\sharp}$ is given as in Tables 4 , 5 , and 6 , respectively.

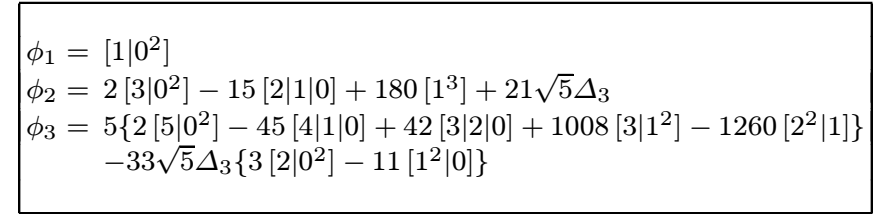

TABLE 4. Canonical invariant basis for $G_{H_{3}}$.

We explain how to determine these canonical invariant bases. We pick out the case $\sharp=F_{4}$ as an example. The remaining cases $\sharp=H_{3}, H_{4}$ can be treated in a similar manner. We begin with an invariant basis constructed by Mehta [Mehta 88]. As an invariant basis for the group $G_{F_{4}}$, he has given $\left(\psi_{1}, \psi_{2}, \psi_{3}, \psi_{4}\right)=\left(I_{2}, I_{6}, I_{8}, I_{12}\right)$, where

$$
I_{2 k}=\left(8-2^{2 k-1}\right) S_{2 k}+\sum_{i=1}^{k-1}\left(\begin{array}{c}
2 k \\
2 i
\end{array}\right) S_{2 i} S_{2(k-i)}
$$

$$
(k=1,3,4,6)
$$

with $S_{m}=x_{1}^{m}+x_{2}^{m}+x_{3}^{m}+x_{4}^{m}$. Since the degrees $\left(d_{1}, d_{2}, d_{3}, d_{4}\right)=(2,6,8,12)$ are mutually distinct, the invariant basis $\left(\psi_{1}, \psi_{2}, \psi_{3}, \psi_{4}\right)$ is an orthogonal system relative to the inner product $(3-1)$. To determine the canonical invariant basis $\left(\phi_{1}, \phi_{2}, \phi_{3}, \phi_{4}\right)$, we try to solve the system of partial differential equations (3-2). Taking the degrees into account, we can find its solution in the form

$$
\left\{\begin{array}{l}
\phi_{1}=\psi_{1} \\
\phi_{2}=\psi_{2}+a_{1} \psi_{1}^{3} \\
\phi_{3}=\psi_{3}+a_{2} \psi_{2} \psi_{1}+a_{3} \psi_{1}^{4} \\
\phi_{4}=\psi_{4}+a_{4} \psi_{3} \psi_{1}^{2}+a_{5} \psi_{2}^{2}+a_{6} \psi_{2} \psi_{1}^{3}+a_{7} \psi_{1}^{6}
\end{array}\right.
$$

with some constants $a_{1}, a_{2}, \ldots, a_{7}$. The existence of such constants is guaranteed theoretically. But we must determine them explicitly. Substituting (4-1) into (3-2), we obtain a system of linear equations for $a_{1}, a_{2}, \ldots, a_{7}$. By solving it, $\left(\phi_{1}, \phi_{2}, \phi_{3}, \phi_{4}\right)$ can be determined explicitly. This procedure is quite elaborate and requires computerassisted calculations (we use Maple for this purpose). Determining $a_{1}, a_{2}, \ldots, a_{7}$ in this manner, we are able to

$$
\begin{aligned}
\phi_{1}= & {\left[1 \mid 0^{3}\right] } \\
\phi_{2}= & {\left[3 \mid 0^{3}\right]-5\left[2|1| 0^{2}\right]+30\left[1^{3} \mid 0\right] } \\
\phi_{3}= & 3\left[4 \mid 0^{3}\right]-28\left[3|1| 0^{2}\right]+98\left[2^{2} \mid 0^{2}\right]-84\left[2\left|1^{2}\right| 0\right]+1512\left[1^{4} \mid 0\right] \\
\phi_{4}= & {\left[6 \mid 0^{3}\right]-22\left[5|1| 0^{2}\right]+143\left[4|2| 0^{2}\right]+66\left[4\left|1^{2}\right| 0\right]-308\left[3^{2} \mid 0^{2}\right] } \\
& +308[3|2| 1 \mid 0]-5544\left[3 \mid 1^{3}\right]-2310\left[2^{3} \mid 0\right]+4620\left[2^{2} \mid 1^{2}\right]
\end{aligned}
$$

TABLE 5. Canonical invariant basis for $G_{F_{4}}$. 


\begin{tabular}{|c|c|}
\hline$\phi_{1}=$ & {$\left[1 \mid 0^{3}\right]$} \\
\hline$\phi_{2}=$ & $\begin{array}{l}{\left[6 \mid 0^{3}\right]-22\left[5|1| 0^{2}\right]+99\left[4|2| 0^{2}\right]+198\left[4\left|1^{2}\right| 0\right]-176\left[3^{2} \mid 0^{2}\right]} \\
-66[3|2| 1 \mid 0]-4752\left[3 \mid 1^{3}\right]-330\left[2^{3} \mid 0\right]+3960\left[2^{2} \mid 1^{2}\right]-462 \sqrt{5} \Delta_{4}\end{array}$ \\
\hline$\phi_{3}=$ & $\begin{array}{l}3\left[10 \mid 0^{3}\right]-190\left[9|1| 0^{2}\right]+2907\left[8|2| 0^{2}\right]+5814\left[8\left|1^{2}\right| 0\right]-14820\left[7|3| 0^{2}\right] \\
-63270[7|2| 1 \mid 0]+61560\left[7 \mid 1^{3}\right]+31122\left[6|4| 0^{2}\right]+238602[6|3| 1 \mid 0] \\
+414960\left[6\left|2^{2}\right| 0\right]-311220\left[6|2| 1^{2}\right]-36556\left[5^{2} \mid 0^{2}\right]-204516[5|4| 1 \mid 0] \\
-1110018[5|3| 2 \mid 0]-3361176\left[5|3| 1^{2}\right]+5913180\left[5\left|2^{2}\right| 1\right] \\
+1934010\left[4^{2}|2| 0\right]+6802380\left[4^{2} \mid 1^{2}\right]-62244\left[4\left|3^{2}\right| 0\right] \\
-3267810[4|3| 2 \mid 1]-28009800\left[4\left|2^{3}\right| 0\right]+5228496\left[3^{3} \mid 1\right] \\
+17428320\left[3^{2} \mid 2^{2}\right]+1254 \sqrt{5} \Delta_{4}\left\{15\left[4 \mid 0^{3}\right]\right. \\
\left.-76\left[3|1| 0^{2}\right]+158\left[2^{2} \mid 0^{2}\right]-24\left[2\left|1^{2}\right| 0\right]+4032\left[1^{4}\right]\right\}\end{array}$ \\
\hline$\phi_{4}=$ & $\begin{array}{l}2\left[15 \mid 0^{3}\right]-290\left[14|1| 0^{2}\right]+10962\left[13|2| 0^{2}\right] \\
+21924\left[13\left|1^{2}\right| 0\right]-160080\left[12|3| 0^{2}\right]-580725[12|2| 1 \mid 0] \\
-156600\left[12 \mid 1^{3}\right]+1124562\left[11|4| 0^{2}\right]+6347172[11|3| 1 \mid 0] \\
+10445220\left[11\left|2^{2}\right| 0\right]+2401200\left[11|2| 1^{2}\right]-4011338\left[10|5| 0^{2}\right] \\
-39631806[10|4| 1 \mid 0]-74993478[10|3| 2 \mid 0]+93454704\left[10|3| 1^{2}\right] \\
-163041480\left[10\left|2^{2}\right| 1\right]+6894112\left[9|6| 0^{2}\right]+153571414[9|5| 1 \mid 0] \\
+234767325[9|4| 2 \mid 0]-789274440\left[9|4| 1^{2}\right]+487555656\left[9\left|3^{2}\right| 0\right] \\
+361940880[9|3| 2 \mid 1]+3353275800\left[9 \mid 2^{3}\right]-3877938\left[8|7| 0^{2}\right] \\
-350953389[8|6| 1 \mid 0]-414939366[8|5| 2 \mid 0]+2156133528\left[8|5| 1^{2}\right] \\
-1281658509[8|4| 3 \mid 0]+1977748380[8|4| 2 \mid 1]-2823138864\left[8\left|3^{2}\right| 1\right] \\
-11401137720\left[8|3| 2^{2}\right]+465352560\left[7^{2}|1| 0\right]+219749820[7|6| 2 \mid 0] \\
-1551175200\left[7|6| 1^{2}\right]+3221273832[7|5| 3 \mid 0]-13029871680[7|5| 2 \mid 1] \\
+116338140\left[7\left|4^{2}\right| 0\right]+5584230720[7|4| 3 \mid 1]+44208493200\left[7|4| 2^{2}\right] \\
+4343290560\left[7\left|3^{2}\right| 2\right]-4234708296\left[6^{2}|3| 0\right]+23526157200\left[6^{2}|2| 1\right] \\
-218457174[6|5| 4 \mid 0]-7528370304[6|5| 3 \mid 1]-42347082960\left[6|5| 2^{2}\right] \\
+9074374920\left[6\left|4^{2}\right| 1\right]-70578471600[6|4| 3 \mid 2]+237143664576\left[6 \mid 3^{3}\right] \\
+739393512\left[5^{3} \mid 0\right]-4436361072\left[5^{2}|4| 1\right]+186327165024\left[5^{2}|3| 2\right] \\
-33272708040\left[5\left|4^{2}\right| 2\right]-186327165024\left[5|4| 3^{2}\right]+299454372360\left[4^{3} \mid 3\right] \\
+957 \sqrt{5} \Delta_{4}\left\{105\left[9 \mid 0^{3}\right]-1827\left[8|1| 0^{2}\right]+8661\left[7|2| 0^{2}\right]+32167\left[7\left|1^{2}\right| 0\right]\right. \\
-14937\left[6|3| 0^{2}\right]-136078[6|2| 1 \mid 0]-721221\left[6 \mid 1^{3}\right]+7350\left[5|4| 0^{2}\right] \\
+219203[5|3| 1 \mid 0]+408861\left[5\left|2^{2}\right| 0\right]+2676017\left[5|2| 1^{2}\right]-204250\left[4^{2}|1| 0\right] \\
-335350[4|3| 2 \mid 0]-1700975\left[4|3| 1^{2}\right]-9676225\left[4\left|2^{2}\right| 1\right] \\
\left.+695970\left[3^{3} \mid 0\right]+8701810\left[3^{2}|2| 1\right]+7709820\left[3 \mid 2^{3}\right]\right\}\end{array}$ \\
\hline
\end{tabular}

TABLE 6. Canonical invariant basis for $G_{H_{4}}$.

obtain the result in Table 5 after suitable renormalizations; recall that the canonical invariant polynomials are unique only up to nonzero constant multiples.

Also in the cases $\sharp=H_{3}, H_{4}$, the same procedures as explained above with the invariant bases constructed by Mehta [Mehta 88] lead to the results in Tables 4 and 6.

\section{MEAN VALUE PROBLEM}

The proof of Theorem 2.1 consists of verifying the criterion (3-4) by using the explicit formulas for the canonical invariant bases obtained in Section 4 . If $F(k)$ is a fundamental region for the action of $G$ on $P(k)$, then the criterion (3-4) is equivalent to the nonvanishing of the multiple integrals:

$$
I_{i}(k)=\int_{F(k)} \phi_{i}(x) d x \quad(i=1, \ldots, n) .
$$

If $P$ is a regular convex polytope in $\mathbb{R}^{n}$, then one can take a fundamental region $F(k)$ in the following manner: Take a sequence $P_{0}, P_{1}, \ldots, P_{n}$ of regular polytopes with $P_{n}=$ $P$ such that $P_{i}$ is a face of $P_{i+1}$ for each $i \in\{0,1, \ldots, n-$ 1 ). Denote by $p_{i}$ the center of $P_{i}$ (note that $p_{n}=0$ ). Let $F(k)$ be the $k$-simplex having $p_{0}, p_{1}, \ldots, p_{k}$ as its vertices. 


\begin{tabular}{|c|l|}
\hline \multirow{2}{*}{3,5} & $p_{0}=(\tau, 1,0)$ \\
& $p_{1}=(\tau, 0,0)$ \\
& $p_{2}=\frac{\tau^{2}}{3}\left(\tau, 0, \tau^{-1}\right)$ \\
\hline \multirow{4}{*}{5,3} & $p_{0}=\left(\tau, 0, \tau^{-1}\right)$ \\
& $p_{1}=(\tau, 0,0)$ \\
& $p_{2}=\frac{\tau}{\sqrt{5}}(\tau, 1,0)$ \\
\hline \multirow{4}{*}{$3,4,3$} & $p_{0}=(1,0,0,1)$ \\
& $p_{1}=\left(\frac{1}{2}, \frac{1}{2}, 0,1\right)$ \\
& $p_{2}=\left(\frac{1}{3}, \frac{1}{3}, \frac{1}{3}, 1\right)$ \\
& $p_{3}=(0,0,0,1)$ \\
$\{3,3,5\}$ & $p_{0}=\left(\tau, 1, \tau^{-1}, 0\right)$ \\
& $p_{1}=(\tau, 1,0,0)$ \\
& $p_{2}=\frac{2 \tau}{3}\left(\tau, \tau^{-1}, 0,0\right)$ \\
& $p_{3}=\frac{\tau^{2}}{4}\left(\tau^{2}, 1,0, \tau^{-2}\right)$ \\
\hline \multirow{5}{*}{$5,3,3$} & $p_{0}=\left(\tau^{2}, 1,0, \tau^{-2}\right)$ \\
& $p_{1}=\tau\left(\tau, \tau^{-1}, 0,0\right)$ \\
& $p_{2}=\frac{2 \tau}{\sqrt{5}}(\tau, 1,0,0)$ \\
& $p_{3}=\frac{\tau^{2}}{2}\left(\tau, 1, \tau^{-1}, 0\right)$ \\
\hline
\end{tabular}

TABLE 7. Vertices of characteristic simplices.

It is easy to see that $F(k)$ becomes a fundamental region for the action of $G$ on $P(k)$. The simplex $F(n-1)$ is called the characteristic simplex of $P$ in [Coxeter 73].

For each exceptional regular convex polytope, the vertices $p_{0}, p_{1}, \ldots, p_{n}$ of its characteristic simplex are given as in Table 7. With these data, using Maple, we can evaluate the multiple integrals $I_{i}(k)$ and check that they do not vanish (see [Iwasaki et al. 01] for full details). This implies that the criterion (3-4) of Theorem 3.2 is verified and therefore Theorem 2.1 is established. Without computer assistance, the proof presented here would not have been possible. We wonder whether there exists a more conceptual proof of the theorem.

\section{REFERENCES}

[Beckenbach and Reade 43] E. F. Beckenbach and M. Reade, "Mean values and harmonic polynomials", Trans. Amer. Math. Soc. 53 (1943), 230-238.

[Beckenbach and Reade 45] E. F. Beckenbach and M. Reade, "Regular solids and harmonic polynomials", Duke Math. J. 12 (1945), 629-644.

[Coxeter 73] H. M. S. Coxeter, Regular polytopes, 3rd ed., Dover, New York, 1973.

[Flatto 61] L. Flatto, "Functions with a mean value property", J. Math. Mech. 10 (1961), 11-18.
[Flatto 63] L. Flatto, "Functions with a mean value property, II", Amer. J. Math. 85 (1963), 248-270.

[Flatto and Wiener 69] L. Flatto and Sister M. M. Wiener, "Invariants of finite reflection groups and mean value problems", Amer. J. Math. 91 (1969), 591-598.

[Flatto and Wiener 70] L. Flatto and Sister M. M. Wiener, "Regular polytopes and harmonic polynomials", Canad. J. Math. 22 (1970), 7-21.

[Friedman 1957] A. Friedman, "Mean-values and polyharmonic polynomials", Michigan Math. J. 4 (1957), 67-74.

[Haeuslein 70] G. K. Haeuslein, "On the algebraic independence of symmetric functions", Proc. Amer. Math. Soc. 25 (1970), 179-182.

[Humphreys 90] J. E. Humphreys, Reflection groups and Coxeter groups, Cambridge Univ. Press, Cambridge, 1990

[Iwasaki 97a] K. Iwasaki, "Polytopes and the mean value property", Discrete \& Comput. Geometry 17 (1997), 163-189.

[Iwasaki 97b] K. Iwasaki, "Regular simplices, symmetric polynomials, and the mean value property", J. Analyse Math. 72 (1997), 279-298.

[Iwasaki 97c] K. Iwasaki, "Basic invariants of finite reflection groups", J. Algebra 195 (1997), 538-547.

[Iwasaki 99a] K. Iwasaki, "Invariants of finite reflection groups and the mean value problem for polytopes", Bull. London Math. Soc. 31 (1999), 477-483.

[Iwasaki 99b] K. Iwasaki, "Triangle mean value property", Aequationes Math. 57 (1999), 206-220.

[Iwasaki 00] K. Iwasaki, "Recent progress in polyhedral harmonics", Acta Applicandae Math. 60 (2000), 179-197.

[Iwasaki et al. 01] K. Iwasaki, A. Kenma and K. Matsumoto, "Polynomial invariants and harmonic functions related to exceptional regular polytopes", Kyushu Univ. Preprint Series in Math. 2000-11, Kyushu Univ., Fukuoka (2000), 15 pages.

[Kakutani and Nagumo 35] S. Kakutani and M. Nagumo, "On the functional equation $\sum_{\nu=0}^{n-1} f\left(z+e^{2 \nu \pi i / n} \xi\right)=$ $n f(z)$ " (in Japanese), Zenkoku Sûgaku Danwakai 66 (1935), 10-12.

[Mehta 88] M. L. Metha, "Basic sets of invariant polynomials for finite reflection groups", Comm. Algebra 16 (1988), 1083-1098.

[Steinberg 64] R. Steinberg, "Differential equations invariant under finite reflection groups", Trans. Amer. Math. Soc. 112 (1964), 392-400. 
[Walsh 36] J. L. Walsh, "A mean value theorem for polynomials and harmonic polynomials", Bull. Amer. Math. Soc. 42 (1936), 923-930.
[Zalcman 73] L. Zalcman, "Mean values and differential equations", Israel. J. Math. 14 (1973), 339-352.

Katsunori Iwasaki, Kyushu University, 6-10-1 Hakozaki, Higashi-ku, Fukuoka 812-8581 Japan

(iwasaki@math.kyushu-u.ac.jp)

Atsufumi Kenma, Matsue-Minami High School, 1-1-1 Yakumo-dai, Matsue 690-8519 Japan

Keiji Matsumoto, Division of Mathematics, Graduate School of Science, Hokkaido University, Kita 10 Nishi 8, Kita-ku, Sapporo 060-0810 Japan (matsu@math.sci.hokudai.ac.jp)

Received June 21,2001; accepted in revised form March 31, 2002. 